\title{
Reduced-Order Model for an Impacting Beam using the Karhunen-Loève Expansion
}

C. WOLTER ${ }^{1}$, M.A. TRINDADE, R. SAMPAIO, Department of Mechanical Engineering, Pontifícia Universidade Católica do Rio de Janeiro, 22453-900 Rio de Janeiro, RJ, Brasil.

\begin{abstract}
The Karhunen-Loève expansion (KLE), also known in the literature as the proper orthogonal decomposition, is a powerful tool for the model reduction of structural systems. Although the method has been used for quite some time in turbulence studies to uncover spatial coherent structures in flow fields, only recently has it been applied to structural dynamics problems. The KL method is a primarily statistical one where the system dynamics is assumed to be a second-order stochastic process. It consists in obtaining a set of orthogonal eigenfunctions where the dynamics is to be projected. Practically, one constructs a spatial autocorrelation tensor and then performs its spectral decomposition. The resulting eigenfunctions will provide the required proper orthogonal modes (POMs) or empirical eigenmodes and the correspondent empirical eigenvalues (or proper orthogonal values, POVs) represent the mean energy contained in that projection. Finally, one uses a number of the computed modes in Galerkin's method in order to obtain a reduced dimension system (this is sometimes called the KLG method). Although this method can also be applied to linear systems, its main application stems from nonlinear ones. In this present work, such a system is studied, namely a linear clamped beam impacting a flexible barrier. The KLE will be used to look into the dynamics of the system and to generate a reduced-order model (ROM).
\end{abstract}

\section{Introduction}

Although there are some engineering systems where impacts are part of the project [3], most of the time this phenomenon is related to wear, fatigue and noise as, for example, in the case of gear boxes. The interest in vibroimpact systems arises due to their intrinsic nonlinear characteristic which prevents their study through more traditional methods such as modal analysis. Actually, systems of this kind have an extremely complex dynamic behaviour, sometimes even chaotic $[3,4,6]$. Therefore, they are normally studied with bifurcation diagrams and Poincaré maps. However, most of the vibroimpact systems investigated so far consists of simple ones with a single degree of freedom [3]. It is expected that the flexibility of a structure will

\footnotetext{
${ }^{1}$ The author gratefully acknowledges the financial support provided by FAPERJ through a graduate scholarship, grant E-26/150.687/2000.
} 
play an important role in its impact response, specially through the excitations of many of its degrees of freedom. This is another motivation for the application of the KL expansion to this problem. Moreover, the fact that the considered system is piecewise linear will allow a direct comparison between the POMs obtained with the KL expansion and the associated linear system mode shapes.

The main objective of this paper will be the construction of a reduced-order model via Galerkin projection, using the POMs obtained with the KL expansion as trial functions. This new model will be compared to the original one that used the first ten mode shapes of a cantilever beam as trial functions.

\section{The Karhunen-Loève Decomposition}

Let a dynamical system be governed by equations whose solutions give a flow, i.e., a function of time and space that describes the evolution of a particular state, denoted by $\mathbf{u}(\mathbf{x}, t)$ and defined on position $\mathbf{x} \in \mathcal{D}$, where $\mathcal{D}$ is a Hilbert space, and time $t \in[0, \infty)$.

\subsection{Main Hypothesis}

In order to define the autocorrelation tensor for the flow, it is modeled as a secondorder stochastic process. However, it is desirable to avoid the mathematical description of the sample space, $\sigma$-algebra and probability measure associated with the flow. A great advantage of the KLE is that this description is unnecessary, though two aditional assumptions are needed: the flow is supposed to be strictsense time-stationary and ergodic [7]. Let $\mathbf{v}(\mathbf{x}, t)$ define the deviation from the mean flow, i.e.,

$$
\mathbf{v}(\mathbf{x}, t)=\mathbf{u}(\mathbf{x}, t)-E[\mathbf{u}(\mathbf{x}, t)] .
$$

Hence, $\mathbf{v}(\mathbf{x}, t)$ is a stochastic process with zero mean and consequently its autocorrelation tensor equals its autocovariance tensor [8]. If $\mathbf{v}(\mathbf{x}, t)$ is real, then the two-point spatial autocorrelation function is defined by the dyadic product

$$
\mathbf{R}\left(\mathbf{x}, \mathbf{x}^{\prime}\right)=E\left[\mathbf{v}(\mathbf{x}, t) \otimes \mathbf{v}\left(\mathbf{x}^{\prime}, t\right)\right] .
$$

A final assumption regarding the flows $\mathbf{u}(\mathbf{x}, t)$ and $\mathbf{v}(\mathbf{x}, t)$ is that they are continuous in quadratic mean, what implies the continuity of $\mathbf{R}\left(\mathbf{x}, \mathbf{x}^{\prime}\right)$ in $\mathcal{D}$ [7].

\subsection{Model Reduction}

In order to find a reduced-order flow model that still reveals the main features contained in the dynamics, one can search for an expansion of the form

$$
\mathbf{v}(\mathbf{x}, t)=\sum_{k} a_{k}(t) \boldsymbol{\psi}_{k}(\mathbf{x}),
$$

with

$$
E\left[a_{k}(t) a_{l}(t)\right]=\lambda_{k} \delta_{k l}
$$


i.e., the modes are uncorrelated, and

$$
\left\langle\boldsymbol{\psi}_{k}, \boldsymbol{\psi}_{l}\right\rangle=\int_{\mathcal{D}} \sum_{j=1}^{n} \psi_{k_{j}}(\mathbf{x}) \psi_{l_{j}}(\mathbf{x}) d \mathbf{x}=\delta_{k l}
$$

meaning that the set $\left\{\boldsymbol{\psi}_{k}\right\} \in \mathcal{L}_{2}^{n}$ is orthonormal. Inserting equation (2.3) into (2.2) and using the relation given by (2.4), one obtains

$$
\mathbf{R}\left(\mathbf{x}, \mathbf{x}^{\prime}\right)=\sum_{k} \lambda_{k} \boldsymbol{\psi}_{k}(\mathbf{x}) \otimes \boldsymbol{\psi}_{k}\left(\mathbf{x}^{\prime}\right)
$$

Since by definition and according to our assumptions, $\mathbf{R}\left(\mathbf{x}, \mathbf{x}^{\prime}\right)$ is a positive semidefinite continuous Hermitian operator, Mercer's Theorem [9] guarantees the existence and uniqueness of the spectral representation of $\mathbf{R}\left(\mathbf{x}, \mathbf{x}^{\prime}\right)$ given by (2.6), where $\left\{\boldsymbol{\psi}_{k}\right\}$ are the eigenfunctions of the integral operator with kernel $\mathbf{R}\left(\mathbf{x}, \mathbf{x}^{\prime}\right)$ and $\left\{\lambda_{k}\right\}$ are the corresponding real and nonnegative eigenvalues so that

$$
\int_{\mathcal{D}} \mathbf{R}\left(\mathbf{x}, \mathbf{x}^{\prime}\right) \boldsymbol{\psi}_{k}\left(\mathbf{x}^{\prime}\right) d \mathbf{x}^{\prime}=\lambda_{k} \boldsymbol{\psi}_{k}(\mathbf{x})
$$

Then, the Karhunen-Loève Theorem [7] states that a continuous second-order stochastic process with autocovariance tensor $\mathbf{K}\left(\mathbf{x}, \mathbf{x}^{\prime}\right)$ can be expanded in a series analogous to (2.3) where $\left\{\boldsymbol{\psi}_{k}\right\}$ are the eigenfunctions of the integral operator with kernel $\mathbf{K}\left(\mathbf{x}, \mathbf{x}^{\prime}\right)$ and $\left\{\lambda_{k}\right\}$ are the corresponding eigenvalues. Since for $\mathbf{v}(\mathbf{x}, t)$ the autocovariance equals its autocorrelation, it is thus proved that the expansion stated in equation (2.3) is realizable. The set $\left\{\boldsymbol{\psi}_{k}\right\}$ is formed by the POMs, also called coherent structures [10].

The original flow $\mathbf{u}(\mathbf{x}, t)$ can, therefore, be reconstructed with reduced dimension through the truncation of the series (2.3) and addition of the mean flow:

$$
\mathbf{u}(\mathbf{x}, t)=\sum_{k=1}^{K} a_{k}(t) \boldsymbol{\psi}_{k}(\mathbf{x})+E[\mathbf{u}(\mathbf{x}, t)]
$$

where the temporal coeficients $a_{k}(t)$ are easily found by projecting the flow onto each POM $\boldsymbol{\psi}_{k}(\mathbf{x})$, i.e.,

$$
a_{k}(t)=\left\langle\mathbf{v}(\mathbf{x}, t), \boldsymbol{\psi}_{k}(\mathbf{x})\right\rangle .
$$

Finally, the eigenvalues may be written, using the ergodic hypothesis, as

$$
\lambda_{k}=\left\langle\boldsymbol{\psi}_{k}, \mathbf{R} \boldsymbol{\psi}_{k}\right\rangle=E\left[\left|\left\langle\boldsymbol{\psi}_{k}, \mathbf{v}\right\rangle\right|^{2}\right]=\lim _{T \rightarrow \infty} \frac{1}{T} \int_{0}^{T}\left|\left\langle\boldsymbol{\psi}_{k}, \mathbf{v}\right\rangle\right|^{2} d t
$$

indicating that they are a measure of the system mean energy projected on the axis $\psi_{k}$ in the functional space. Besides, it can be shown that the total mean energy equals the sum of all eigenvalues and that it is optimal in the sense that given a fixed number of modes, no other linear decomposition can contain as much energy as the KLE for the particular dynamics from which the POMs were calculated [10]. 


\section{Pratical Construction of POMs}

There are two pratical methods available for the construction of the KLE, namely the original direct method and the more recent snapshot method introduced in [10]. Since this work deals with the direct method, it is the only one discussed.

\subsection{Direct Method}

In this method, the displacements of a dynamical system are measured or calculated at $\mathrm{N}$ locations and labeled $u_{1}(t), u_{2}(t), \ldots, u_{N}(t)$. Sampling these displacements $M$ times, we can form the following $M \times N$ ensemble matrix:

$$
\mathbf{U}=\left[\begin{array}{llll}
\mathbf{u}_{1} & \mathbf{u}_{2} & \ldots & \mathbf{u}_{N}
\end{array}\right]=\left[\begin{array}{cccc}
u_{1}\left(t_{1}\right) & u_{2}\left(t_{1}\right) & \ldots & u_{n}\left(t_{1}\right) \\
\vdots & \vdots & \ddots & \vdots \\
u_{1}\left(t_{M}\right) & u_{2}\left(t_{M}\right) & \ldots & u_{n}\left(t_{M}\right)
\end{array}\right]
$$

Thus, using stationarity and ergodicity hypothesie, the variation from the mean is

$$
\mathbf{V}=\mathbf{U}-\frac{1}{M}\left[\begin{array}{cccc}
\sum_{i=1}^{M} u_{1}\left(t_{i}\right) & \sum_{i=1}^{M} u_{2}\left(t_{i}\right) & \ldots & \sum_{i=1}^{M} u_{N}\left(t_{i}\right) \\
\vdots & \vdots & \ddots & \vdots \\
\sum_{i=1}^{M} u_{1}\left(t_{i}\right) & \sum_{i=1}^{M} u_{2}\left(t_{i}\right) & \ldots & \sum_{i=1}^{M} u_{N}\left(t_{i}\right)
\end{array}\right]
$$

and the spatial correlation matrix of dimension $N \times N$ formed as

$$
\mathbf{R}=\frac{1}{M} \mathbf{V}^{T} \mathbf{V}
$$

The POMs are then given by the eigenvectors of $\mathbf{R}$, orthogonal due to its simmetry. Eigenvalues will provide the POVs. Clearly, the matrix dimension is determined by the number of sampling points $N$. For a three-dimensional flow $\mathbf{v}(\mathbf{x}, t) \in \mathbb{R}^{3}$, the number of operations needed for the diagonalization of $\mathbf{R}$ is $\mathcal{O}\left(N^{3}\right)$ [2].

\section{Modeling the Vibroimpact System}

Consider the cantilever steel beam depicted in Figure 1. Its transversal displacement $w(x, t)$ is constrained in a section starting at the free end by two elastic barriers with $50 \mathrm{~mm}$ of length and located at a distance $\varepsilon$ from the top and bottom surfaces of the beam. The beam geometric properties are: length $L=510 \mathrm{~mm}$, thickness $h=5.25 \mathrm{~mm}$ and width $b=24.85 \mathrm{~mm}$. The elastic barrier modeling was performed through a discretization that considers the existence of linear springs spaced by $0.1 \mathrm{~mm}$ sections, so that the system forced equation of motion is, according to Euler-Bernoulli theory and at first disconsidering structural damping:

$$
E I \frac{\partial^{4} w(x, t)}{\partial x^{4}}+\rho A \frac{\partial^{2} w(x, t)}{\partial t^{2}}=F_{f} \sin \left(\omega_{f} t\right) \delta\left(x-x_{f}\right)+\sum_{i=1}^{N} F_{c i}(t) \delta\left(x-x_{c i}\right)
$$




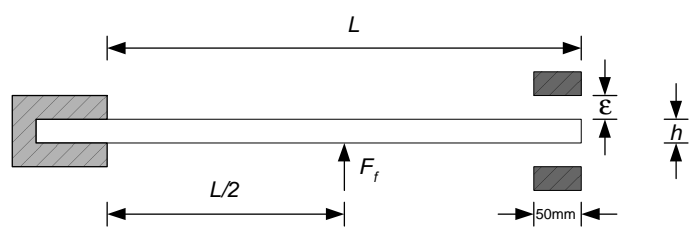

Figure 1: Vibroimpact system.

where $F_{f}$ is the sinusoidal forcing magnitude applied at $x_{f}=L / 2, \omega_{f}$ its frequency, $\delta$ is the Dirac delta function, $x_{c i}$ the positions where the barriers were discretized, i.e., assumed positions of the springs (here $x_{c i}=\{460 ; 460.1 ; \ldots 510\} \mathrm{mm}$ ), and $F_{c i}$ the respective forces acting on the beam due to its impact against the springs. These impact forces assume the following values:

$$
F_{c i}(t)= \begin{cases}0, & \left|w\left(x_{c i}, t\right)\right| \leq \varepsilon \\ -k\left[w\left(x_{c i}, t\right)-\varepsilon \operatorname{sign}\left(w\left(x_{c i}, t\right)\right)\right], & \left|w\left(x_{c i}, t\right)\right|>\varepsilon\end{cases}
$$

where $k$ represents the spring stiffness that was taken as $10^{9} \mathrm{~N} / \mathrm{m}$.

The PDE (4.1) discretization was performed via Galerkin's method using as trial functions the first ten mode shapes belonging to the associated linear system [5]. An approximate solution of the form $\hat{w}(x, t)=\sum_{i} q_{i}(t) \phi_{i}(x)$ was sought with

$$
\phi_{i}(x)=\cosh \beta_{i} x-\cos \beta_{i} x-\frac{\operatorname{senh} \beta_{i} L-\operatorname{sen} \beta_{i} L}{\cosh \beta_{i} L+\cos \beta_{i} L}\left(\operatorname{senh} \beta_{i} x-\operatorname{sen} \beta_{i} x\right),
$$

where $\beta_{i}$ is an appropriate solution to $\cos \beta L \cosh \beta L=1$, and $q_{i}$ are the temporal coordinates.

\subsection{Damping}

Modal damping ratios were afterwards added to the discretized system of ODEs. The first six modal damping ratios calculated from the frequency response obtained with an experimental setup [11] are $\zeta_{1}=0.0461, \zeta_{2}=0.0084, \zeta_{3}=0.0039, \zeta_{4}=$ $0.0028, \zeta_{5}=0.0023, \zeta_{6}=0.0063$. The other four ratios were assumed as 0.003 .

\subsection{Simulation}

The beam was subjected to a harmonic forcing function with $F_{f}=140 \mathrm{~N}$ and $\omega_{f}=100 \mathrm{rad} / \mathrm{s}$ which is slightly inferior to the beam first natural frequency. The discrete system was integrated using MATLAB ${ }^{\circledR}$ ode 45 th/5th order Runge-Kutta algorithm.

Figure 2 presents the simulation results for the beam free end. The curves represent the top and bottom displacements of the free end between 0 and $0.07 \mathrm{~s}$ and the thick horizontal lines represent the barrier position. 


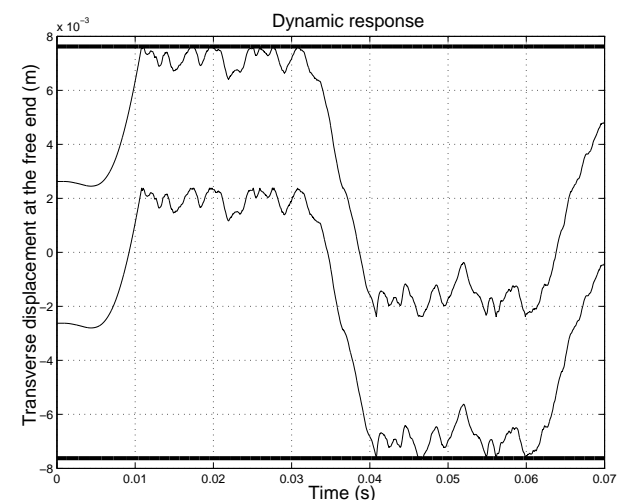

Figure 2: Dynamic response.

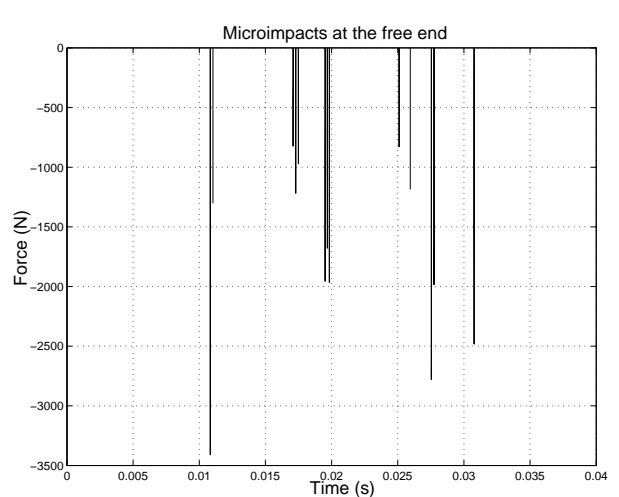

Figure 3: Microimpacts at the free end.

One can easily observe the presence of high frequency oscillations. Actually, in the beginning, the beam movement almost resembles the first mode shape since the excitation frequency is close to the first natural frequency. However, after the first impact and due to the system flexibility, higher modes are excited. These new oscillations have the effect of generating multiple impacts in a very short time interval. These microimpacts depicted in Figure 3 add a lot of complexity to the dynamics.

\section{Application of the Decomposition}

In order to calculate the empirical modes to this problem, the beam dynamics was simulated until a final time of $30 \mathrm{~s}$. The displacements of points spaced by $1 \mathrm{~mm}$ were sampled each $0.03 \mathrm{~s}$. Hence, for each point there were 1000 time samples. For linear systems it is a good strategy to keep the sampling interval greater than the first natural period [12]. In the present case, though, this condition can be somewhat relaxed as the impacts tend to uncorrelate the samples. The KarhunenLoève expansion was then obtained through the direct method.

Figure 4 presents the first 50 POVs. As it can be seen, most of the energy is concentrated in the first POV which accounts for $98.7 \%$ of it. The second POV accounts for only $1.17 \%$ and the remaining ones account for less than $0.1 \%$ each. Furthermore, it is clear that only the first 10 POVs have physical meaning since the other POVs have magnitudes inferior to MATLAB ${ }^{\circledR}$ numerical precision. This is a direct consequence of the fact that only 10 mode shapes were used in the simulation.

Figure 5 shows the first eight empirical eigenmodes and mode shapes for the system. One can see that the first POM significantly differs from the first mode shape reflecting the influence of the barrier upon the system. And while the second POM still presents some difference from the second mode shape, the remaining POMs that are almost indistinguishable from the respective mode shapes. 


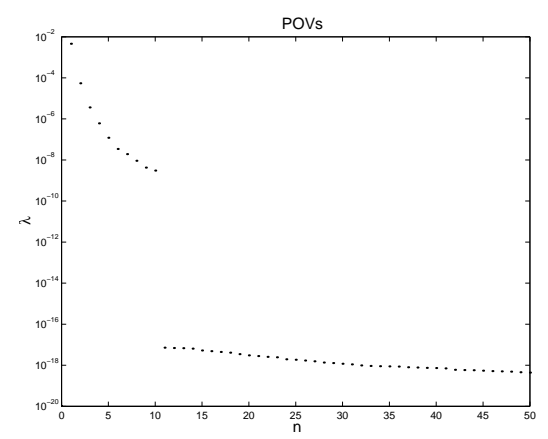

Figure 4: POVs obtained for the vibroimpact problem.
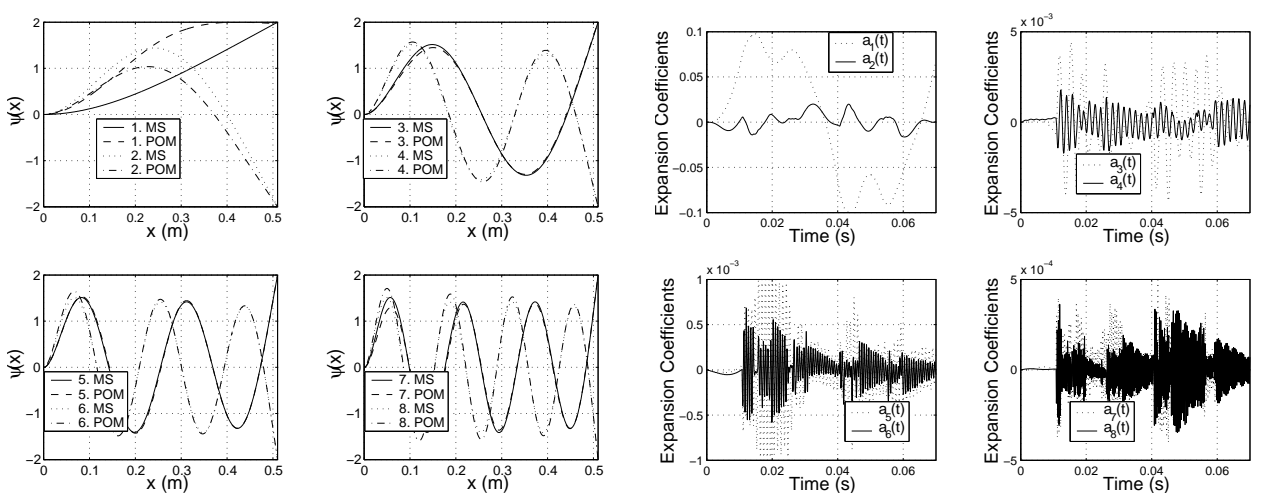

Figure 5: Comparison between empirical eigenmodes and mode shapes.

Figure 6: First eight temporal coefficients for the KL expansion.

The projection of the dynamics onto the POMs provides the temporal coefficients that complete the expansion of the terms accounting for the variation in relation to the mean in equation (2.8). Figure 6 depicts the first eight temporal coefficients related to the POMs of Figure 5. It is interesting to note that at first only the first two POMs account for the dynamics. However, after the first impact, all empirical modes are excited. Besides, the following microimpacts continuously redistribute the energy among the POMs.

Finally, the system dynamics was reconstructed according to the KLE. Figure 7 presents this reconstruction using one, two and three POMs. One can observe that although the first POM has almost $99 \%$ of the energy, the reconstruction based exclusively in this mode is not enough accurate. On the other hand, using three POMs one can achieve an excelent precision in this reconstruction. This result leads to the belief that using these three POMs as trial functions in the Galerkin's method shall yield an accurate reduced-order model. 


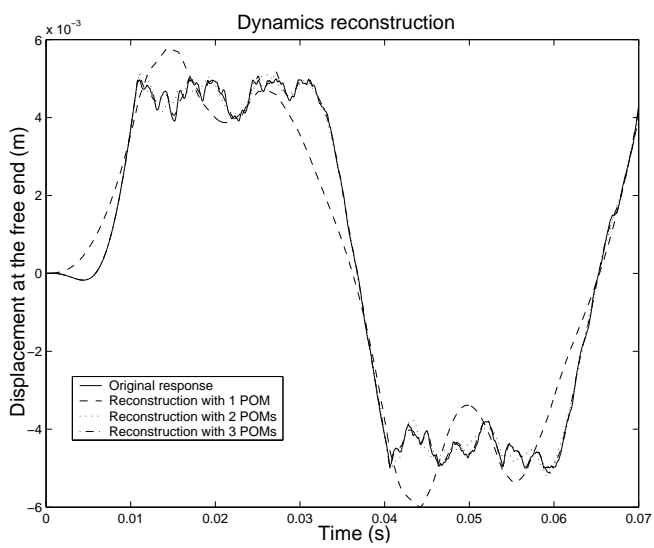

Figure 7: Dynamics reconstruction using one, two and three empirical modes.

\section{Reduced-order Model Formulation}

Rewriting the beam equation of motion (4.1) in a more compact form as

$$
\rho A \frac{\partial^{2} w(x, t)}{\partial t^{2}}=F(x, t)-D(w(x, t)),
$$

where $D(w(x, t))=E I \partial^{4} w(x, t) / \partial x^{4}$, and noting that the use of POMs leads to the following approximate solution

$$
\hat{w}(x, t)=\sum_{i=1}^{n} a_{i}(t) \psi_{i}(x)+E[w(x, t)],
$$

the application of Galerkin's method to the PDE (6.2) using the POMs as trial functions yields this ODE system:

$$
\begin{aligned}
\rho A \ddot{a}_{j}(t)=\int_{0}^{L} F(x, t) \psi_{j}(x)-\sum_{i=1}^{n} a_{i}(t) \int_{0}^{L} D\left(\psi_{i}(x)\right) \psi_{j}(x) d x- \\
\\
\int_{0}^{L} D(E[w(x, t)]) \psi_{j}(x) d x .
\end{aligned}
$$

Since structural damping is not part of the PDE defining the equation of motion and was introduced in the original discretized model a posteriori, the question of how to introduce it in this new model arises at this point. As shown in Figure 5, with the exception of the first POM, all the other empirical modes are very similar to the respective mode shapes. Hence, it was decided to use the same damping ratios from the original model in this new one. Figure 8 presents a comparison between the original and the reduced-order model constructed with 5 POMs. The result is clearly not as good as expected, even though only 3 POMs were required to perform an excellent reconstruction of the dynamics as depicted in Figure 7. 


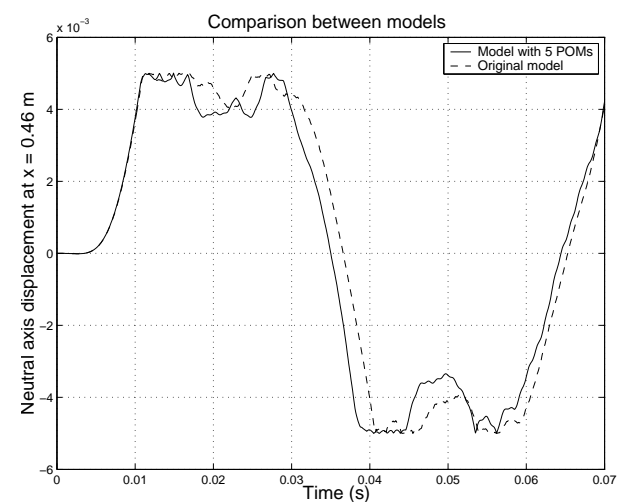

Figure 8: ROM with 5 POMs.

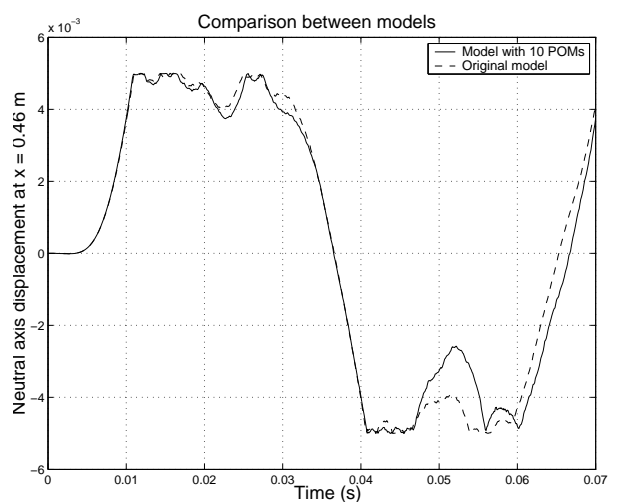

Figure 9: ROM with 10 POMs..

Likewise, Figure 9 shows a comparison between original and the full KLG models. Although the result has improved, one can see that the full KLG model is not yet capable of reproducing the original response, specially regarding low frequency oscillations. A probable explanation for this result is that the use of the modal damping ratios for the first and second POMs is innapropriate as they are physically different. Therefore, good results could be expected if the modal damping ratios were projected onto the base generated by the POMs. In other words, since the first POM does not correspond to any mode shape, actually consisting in a weighted average of them, its damping ratio should also be a weighted average of the modal ratios. This question will be further addressed in a future paper.

\section{Conclusions}

This work presented the application of the KLE to a vibroimpact piecewise linear system. It was shown that the used model is capable of reproducing the existence of microimpacts that have significant effects upon the global system dynamics. Moreover, the empirical eigenmodes and respective temporal coefficients may enhance the understanding of the energy flux throughout the system. Since the original model contained only ten mode shapes, the KL expansion was only able to capture ten POMs. In spite of that, only three were necessary for a good dynamic representation of the system. Finally, a reduced-order model was constructed via Galerkin projection having the POMs as trial functions. Unfortunately this was not good enough due to the use of modal dampings for the empirical modes as previously discussed. This could have been avoided if the structural damping had been accounted for in the PDE through the insertion of a term $c \partial w(x, t) / \partial t$ as proposed in [1]. In this case, though, there would be the additional difficulty of estimating the coefficient $c$ from the experimentally calculated modal damping ratios. It is yet important to highlight that a KLG model can be directly derived from an experiment. 
Resumo A expansão de Karhunen-Loève (KLE), também conhecida na literatura como decomposição ortogonal própria é uma poderosa ferramenta estatística para formulação de modelos reduzidos de estruturas. Para isso, a dinâmica do sistema é modelada como um processo estocástico de segunda ordem e é construído o tensor autocorrelação espacial deste processo, cuja decomposição espectral fornecerá um conjunto de autofunções ortogonais chamados de modos ortogonais próprios (POMs) ou modos empíricos e um conjunto de autovalores associados representando a energia de cada modo. Estes modos empíricos são usados como funções de aproximação no método de Galerkin para se construir um modelo reduzido. Neste trabalho, será estudado um sistema de vibroimpacto linear por partes constituído por uma viga cuja vibração transversal é limitada por batentes elásticos.

\section{References}

[1] M.F.A. Azeez and A.F. Vakakis, Proper orthogonal decomposition (POD) of a class of vibroimpact oscillations, J. Sound Vib., 240, No. 5 (2001), 859-889.

[2] K.S. Breuer and L. Sirovich, The use of the Karhunen-Loève procedure for the calculation of linear eigenfunctions, J. Comput. Phys., 96 (1991), 277-296.

[3] J.P. Cusumano and B.-Y. Bai, Period-infinity periodic motions, chaos, and spatial coherence in a 10 degree of freedom impact oscillator, Chaos, Solit. Frac., 3, No. 5 (1993), 515-535.

[4] M.I. Friswell, J.E.T. Penny and S.D. Garvey. The application of the IRS and balanced realization methods to obtain reduced models of structures with local non-linearities, J. Sound Vib., 196, No. 4 (1996), 453-468.

[5] D.J. Inman, "Engineering Vibration", Prentice-Hall, 1996.

[6] G. Luo and J. Xie, Bifurcations and chaos in a system with impacts, Physica D, 148 (2001), 183-200.

[7] A.J. Newman, "Model reduction via the Karhunen-Loeve expansion part I: an exposition", Technical Report 96-32, Institute for Systems Research, 1996.

[8] A. Papoulis, "Probability, Random Variables, and Stochastic Processes", McGraw-Hill, 1991.

[9] F. Riesz and B. Sz.-Nagy, "Functional Analysis", Frederick Ungar, 1955.

[10] L. Sirovich, Turbulence and the dynamics of coherent structures part I: coherent strucutures, Quart. Appl. Math., 45, No. 3 (1987), 561-571.

[11] C. Wolter, "Uma Introdução à Redução de Modelos Através da Expansão de Karhunen-Loève", Master's thesis, Pontifícia Universidade Católica do Rio de Janeiro, Rio de Janeiro, Brasil, 2001.

[12] C. Wolter, M.A. Trindade and R. Sampaio, Obtaining mode shapes through the Karhunen-Loève expansion for distributed-parameter linear systems, in "Proceedings of the $16^{\text {th }}$ Brazilian Congress of Mechanical Engineering: Vibration and Acoustics" (A. Silveira Neto, S.A.G. Oliveira, A.R. Machado and C.R. Ribeiro, eds.), Vol. 10, pp. 444-452, ABCM, Uberlândia, Brasil, 2001. 